\title{
Personas mayores en situaciones de desastre: un análisis desde su experiencia en el incendio de Valparaíso de 2014
}

Older people in disaster situations: An analysis from their experience at the Valparaíso fire of 2014

\author{
Verónica Rubio Aguilar \\ Universidad Santo Tomás, Chile, $\measuredangle$ vrubioa@santotomas.cl \\ [orcid.org/0000-0002-8965-1461]
}

\section{RESUMEN}

Chile es reconocido como un país sometido a diversos desastres socionaturales, siendo de interés indagar cómo los vivencian las personas mayores, debido a que podrían presentar más dificultades que otros grupos para enfrentarlos. En abril de 2014, Valparaíso fue arrasado por un incendio que dejó más de 12.000 damnificados, concentrándose en el cerro La Cruz, el número más alto de personas mayores afectadas. El objetivo de este estudio cualitativo de carácter exploratorio fue indagar cómo las personas mayores significan su experiencia en situaciones de desastre a partir de este siniestro. Los resultados del análisis interpretativo surgido de las entrevistas en profundidad, develaron que identifican condiciones de vulnerabilidad en el contexto que habitan comprendidas como abandono material y emocional, que su capacidad de respuesta ante los desastres la significan como un tránsito que evoluciona desde el miedo a la resiliencia y que valoran sus recursos personales por sobre aquellos otorgados por la institucionalidad pública, en relación a las estrategias que utilizaron para la reestructuración de sus vidas.

PALABRAS CLAVE: Personas mayores, desastres socionaturales, vulnerabilidad, Chile.

\section{ABSTRACT}

Chile is recognized as a country subjected to several socio-natural disasters, and it is of interest to investigate how the elderly experience those, because they could present more difficulties than other groups to face them. In April 2014, Valparaíso was devastated by a fire that left more than 12,000 people affected, concentrating the highest number of elderly 
people affected on La Cruz hill. The objective of this qualitative exploratory study was to investigate how they mean their experience in disaster situations starting from this incident. The results of the interpretative analysis that emerged from the in-depth interviews revealed that they identify conditions of vulnerability in the context they inhabit, including material and emotional abandonment, that their ability to respond to disasters it is seen as a transit that evolves from fear to resilience, and that they value their personal resources over those garanted by public institutions in relation to the strategies they use to restructure their lives.

KEY WORDS: the elderly, socio-natural disasters, vulnerability, Chile.

\section{INTRODUCCIÓN}

América Latina y el Caribe es una de las regiones del mundo más afectadas por desastres socio-naturales. En relación a esto, hasta hace muy poco tiempo, se ponía mayor énfasis en los agentes causantes y menos en las circunstancias de las poblaciones expuestas. Hoy, en cambio, la preocupación se ha ido desplazando hacia las comunidades que sufren los daños, identificando la vulnerabilidad como principal determinante de los perjuicios provocados por los desastres.

El estudio desarrollado por Vargas (2002) advierte que "el 90\% de las víctimas de desastres viven en países en desarrollo, bajo condiciones de pobreza, lo que las empuja a habitar en áreas y viviendas de alto riesgo" (p. 10). Este podría ser el caso de las personas y familias afectadas por el incendio de Valparaíso de 2014, que, de acuerdo a los primeros diagnósticos del Departamento Social de la Gobernación Provincial de Valparaíso en el momento inmediatamente posterior al incendio, habitaban en las partes más altas de los cerros, laderas empinadas, quebradas y fondos de quebradas, situación que las haría propensas a ser afectadas por desastres. Esta descripción es coincidente con lo que plantea el Observatorio de Valparaíso (2015), en su primer reporte de la reconstrucción, luego del incendio, en que declara que "persisten condiciones que podrían hacer que una lluvia fuerte, o una fogata fuera de control genere una nueva tragedia, dada la gran cantidad de viviendas de material ligero ubicadas en zonas de riesgo y rodeadas por bosques" (p. 8). Por tanto, como señala Vargas (2002), "las consecuencias desastrosas son proporcionales a las vulnerabilidades de las comunidades y los territorios” (p. 10).

En particular, Chile es reconocido, nacional e internacionalmente, como un país sometido a diversos y continuos desastres socionaturales, solo entre los años 2014 y 2015 se documentaron las erupciones de los volcanes Villarica (2015) y Calbuco (2015), y el aluvión ocurrido en el norte del país en 2015, sin considerar los desastres que han ocurrido en los años posteriores, dentro de los cuales se encuentran varios mega incendios. La tarde del 12 de abril del año 2014, se produjo el desastre incendiario más grande ocurrido en la historia de la ciudad de Valparaíso, afectando a los cerros La Cruz, Las Cañas, El Litre, El 
Vergel, Mariposas, Ramaditas, Rocuant y Merced. Según el informe del PNUD (2014), "el fuego consumió cerca de 1.000 hectáreas, dejó alrededor de 3.000 viviendas destruidas, además de escuelas e infraestructura pública y comunitaria, 15 personas fallecidas y cerca de 12.500 personas damnificadas" (p. 6). De estas últimas, cerca de quinientas eran personas mayores.

La situación particular que atraviesan las personas mayores damnificadas, como las del incendio de Valparaíso, es de especial interés debido a que según lo expresan Cohen y Ahearn (1989)

(...) son un grupo que en general, suelen tener dificultades para enfrentar los desastres y sus consecuencias. Es usual que las personas mayores queden aisladas de sus sistemas de apoyo y vivan solas, como resultado de esto, con frecuencia tienen miedo de buscar ayuda. Los problemas típicos en este grupo, después de las catástrofes son la depresión y sensación de impotencia, es desafortunado que una respuesta común en algunos adultos mayores, sea la falta de interés en la reconstrucción de su vida (p. 50).

A nivel internacional, las personas mayores han sido reconocidas como sujetos de derecho hace poco tiempo. El primer paso en esta dirección se tomó en la Primera Asamblea Mundial sobre Envejecimiento, Austria 1982, en donde la comunidad internacional, a través de la Organización de Naciones Unidas, aprobó el Plan de Acción Internacional de Viena sobre Envejecimiento, enfocado en problemáticas demográficas propias de los países desarrollados de la década de 1980. Posteriormente como lo expresa el CELADE (2006) siguieron "otros hitos como la aprobación de los Principios de las Naciones Unidas en favor de las Personas de Edad en 1991 y la Segunda Asamblea Mundial sobre Envejecimiento en 2002" (p. 13). Chile ha adoptado progresivamente las prácticas y recomendaciones internacionales, así desde finales de los 90, ha transitado desde un enfoque asistencialista a un enfoque de derechos y protección social, en el año 2012 se comenzó a implementar la política integral de envejecimiento, la cual dentro de sus objetivos generales busca incrementar el bienestar subjetivo de las personas mayores. En este punto, toman sentido las palabras de Paola (2012), quien indica que

(...) no basta que la política social manifieste en su discurso, que garantice los derechos ciudadanos de los mayores, dado que gran parte de las mismas no cumple en los hechos con esa proclama. Más bien, se debe comenzar por reconocer a los adultos mayores, como actores sociales imprescindibles en la construcción colectiva del reconocimiento de sus derechos (p. 22).

Lo antes dicho puede tener como obstáculo algunas visiones que aún persisten en el imaginario colectivo de la sociedad moderna, en que puede efectuarse una equivocada 
percepción de las personas mayores, "a causa de la estigmatización discriminación e invisibilidad que sufren por parte de sus familias y/o la sociedad" (Olivo \& Piña, 2011, p. 21), situación que se debe revertir, visibilizándolas ante la sociedad y ante la política pública, evidenciando su realidad en distintas esferas y situaciones de la vida, empoderándolas y otorgándoles dignidad a partir del reconocimiento de la importancia de sus subjetividades. Sumando a esto, lo que señala SENAMA (2009) respecto de "las inestables condiciones de salud, seguridad económica y bienestar social, enfrentando, además, la subvaloración y discriminación que se expresa en discursos simbólicos y estructuras socioculturales, respecto a la vejez y el envejecimiento" (p. 7). Esto tiene relación con la construcción social que se tiene de ellas, derivada de mitos y estereotipos, que pueden influir en distintos planos de su vida, desde la oferta de políticas públicas y la valorización de su aporte a la sociedad.

En este escenario el presente estudio en su interés de visibilizar la vivencia subjetiva y desmitificar ciertos estereotipos que desvalorizan a la vejez, se situó en el cerro La Cruz, sector donde se registró el mayor número de personas mayores damnificadas en el incendio de Valparaíso de 2014, más de trescientas, de acuerdo a los registros de la Oficina Comunal del Adulto Mayor de la Ilustre Municipalidad de Valparaíso. Esta temática cobra gran relevancia hoy en día, debido a la transición demográfica en etapa avanzada hacia el envejecimiento que se desarrolla en Chile, razón por la que el número de personas mayores afectadas por desastres está destinado a aumentar. Valparaíso es además la región con mayor número de personas mayores en Chile, según lo declara la AMUCH (2017), alcanzando a 17,9\% (p. 9). Por tanto, existe la necesidad de que se adapten las políticas públicas y prácticas gubernamentales, para asegurar que las necesidades de las personas mayores sean analizadas sistemática y continuamente, evitando que este grupo quede excluido.

Poner atención en cómo las personas significan sus vivencias es relevante porque podremos aproximarnos a sus propias formas de interpretar y comprender el mundo y su realidad social. La pretensión, a decir de Gergen, en Arcila (2009), "no es reflejar la realidad, ni crear teorías universales que den cuenta y expliquen los fenómenos que se representan" (p. 43). Lo que interesa es conocer sus historias, creencias, valores y experiencias anteriores, durante y posteriores al desastre, así como el contexto en el que habitan y las interacciones sociales en que participan. Es por esto que el objetivo general de este estudio fue develar los significados que construyen las personas mayores damnificadas del cerro La Cruz, respecto de su experiencia en situaciones de desastre, a partir del incendio ocurrido en Valparaíso el 12 de abril de 2014.

El marco teórico que sustentó esta investigación se organizó de acuerdo a tres contenidos que se revisarán a continuación: Emergencia, desastres socionaturales y vulnerabilidades; teoría de la continuidad, un enfoque sociocultural de la vejez y significados sociales, una comprensión social del conocer. 


\section{Emergencia, desastres socio naturales y vulnerabilidades}

Para adentrarse en el fenómeno de estudio, es necesario distinguir los conceptos de emergencia y desastre. De acuerdo a lo propuesto por López-Ibor (2004) "el impacto de un peligro en un grupo social tiene que ver con los mecanismos y adaptación que la sociedad ha desarrollado para enfrentar los efectos potencialmente destructivos, si estos son efectivos se está frente a una emergencia" (p. 5). En cambio, desastre, según analiza el mismo López-Ibor, desde la definición que propone el glosario de las Naciones Unidas corresponde a una "disrupción grave del funcionamiento social que causa pérdidas amplias humanas, materiales y del medio que supera la capacidad del grupo afectado para afrontarlas solo con sus propios recursos" (p. 5). Al aplicar estos conceptos en el análisis del incendio ocurrido en Valparaíso, es posible identificar que se estuvo frente a un desastre puesto que la población local no habría podido resolver de manera autónoma la demanda de recursos que necesitaron para enfrentar las consecuencias del incendio. Esto según lo informado en medios de prensa locales y nacionales y lo analizado por autoridades regionales en el Plan de recuperación post desastre (PNUD, 2014), "la gravedad del incendio superó la capacidad local para hacer frente al desastre. Siguiendo con lo estipulado en el plan nacional de protección civil, las autoridades municipales recibieron apoyo de los niveles regional y nacional" (p. 13). El alto impacto de este desastre movilizó a organismos especializados, servicios estatales, sociedad civil, voluntarios y a la academia, para implementar estrategias paliativas y de más largo plazo, orientadas primeramente a solidarizar con las poblaciones afectadas y posteriormente a analizar las condiciones de riesgo, con miras a su prevención y solución. En complemento, la magnitud del incendio (1.145 hectáreas consumidas) y el número de damnificados (12.500) llevarían a calificarlo como desastre.

Los desastres pueden clasificarse en dos grupos, los naturales y los tecnológicos o creados por el hombre, es decir, antrópicos (IFRC, 2014). En el caso de un desastre incendiario, éste se puede incluir en ambos grupos debido a que, por factores naturales, tales como los rayos producidos por las tormentas eléctricas, altas temperaturas, la presencia de fuertes vientos, heladas y/o sequías que secan los pastos, puede naturalmente generar una combustión. No obstante, las estadísticas internacionales y nacionales demuestran que un gran porcentaje (alrededor de un 98\%) de los incendios son antrópicos, es decir, provocados por la acción del hombre (Skarwar, 2014). Es por ello que reciben el nombre de desastres socionaturales.

Amaya en Pérez (2013), agrega que "los desastres son procesos históricamente construidos, producto de la acumulación de riesgos y de la vulnerabilidad, relacionados y derivados del tipo de sociedad y de economía que se han ido desarrollando, con el paso del tiempo" (p. 3). En el caso investigado esto puede advertirse en el resumen ejecutivo presentado por la Secretaría Comunal de Planificación (2014), que refiere 
(...) el incendio se extendió descontroladamente favorecido por diversos factores naturales y sociales, entre ellos las condiciones climáticas características de la zona como los intensos vientos; las características geográficas del territorio como quebradas con fuertes pendientes, que dificultan su accesibilidad y favorecen la propagación del fuego; la propia trama urbano-territorial, con limitaciones de conectividad y acceso, la ausencia de vías de evacuación, la ubicación de las viviendas en zonas de riesgos, inadecuados materiales de construcción de las mismas; y la acumulación de basura $y$ otros materiales en las quebradas que actuaron como combustible para avivar el incendio (p. 5).

La Federación Internacional de Sociedades de La Cruz Roja y de la Media Luna Roja (IFRC, 2014), elabora una ecuación que permite comprender los factores que intervienen en una situación de desastre:

\section{$($ VULNERABILIDAD + PELIGRO) $/$ CAPACIDAD $=$ DESASTRE}

Es decir, un peligro combinado con vulnerabilidad y limitado por la capacidad de las personas, para reducir las consecuencias negativas potenciales, daría lugar a un desastre. Así, la vulnerabilidad puede definirse como "la capacidad disminuida de una persona o un grupo de personas para anticiparse, hacer frente y resistir a los efectos de un peligro natural o causado por la actividad humana, y para recuperarse de los mismos" (p. 40). Es un concepto relativo y dinámico. La vulnerabilidad casi siempre se asocia con la pobreza, pero también son vulnerables las personas que viven en aislamiento, inseguridad e indefensión ante riesgos, traumas o presiones (IFRC, 2014).

En complemento, Vargas (2002) señala que la vulnerabilidad ante un desastre, puede entenderse como "la disposición interna a ser afectado por una amenaza" (p. 16). Respecto a esto, existen tres categorías, siendo la primera de ellas la exposición destructiva ante una determinada amenaza, seguida de la incapacidad de reaccionar adecuadamente cuando la amenaza se presenta y, finalmente, la incompetencia para lograr la recuperación de las condiciones normales de vida. Formiga, Prieto y Medus (2009) añaden que "la vulnerabilidad es mayor en los casos en que se considera que la población carece de recursos económicos o tiene ingresos muy limitados, con escasas relaciones sociales y con carencias importantes en su forma de habitar, considerando la vivienda y su entorno" (p. 38). Por su parte, Cutter, Boruff y Lynn (2003) señalan que los desastres

(...) incrementan la vulnerabilidad social al volver a las comunidades más susceptibles de daño, en la medida que profundiza desigualdades sociales previas, relega a las personas a localidades menos seguras y fragiliza los recursos para el enfrentamiento y recuperación de los impactos de la catástrofe (p. 243). 
En el caso específico de las personas mayores, Hartog (2014) señala que existen cuatro razones principales que pueden explicar el aumento de la vulnerabilidad en aquellas frente a los desastres, desde aspectos individuales, de protección, culturales y sociales, a saber:

(...) a) la salud, la movilidad, la vista y el oído; b) la falta de provisión de servicios adecuados para las personas mayores, tanto a diario como en situaciones de emergencia; c) la discriminación por edad, a raíz de la cual se excluye y aísla a las personas mayores, que a menudo ven violentados sus derechos y d) los niveles de pobreza entre las personas mayores, exacerbados por la falta de protección social, mecanismos y oportunidades de subsistencia (p. 5).

Sin embargo, profundizando el análisis, el desarrollo investigativo internacional y nacional del último decenio, da cuenta que la vulnerabilidad de las personas mayores ante un desastre es el resultado de diversos factores, tanto sociodemográficos como medioambientales. A modo ilustrativo, en el estudio de Sánchez y Egea (2011), se señala que la vulnerabilidad en la vejez no es específica de las características demográficas de las personas mayores, sino un producto de la combinación de características e interacciones entre las amenazas, la exposición y las capacidades de afrontamiento en contextos ambientales específicos. No obstante, esta complejidad no debe olvidarse que este colectivo cuenta con un aspecto de gran valor proporcionado precisamente por la edad: "La experiencia acumulada durante el ciclo vital, que es también la experiencia de los riesgos naturales y sociales en la vida adulta, así como la capacidad para afrontarlos a partir de las formas de administrar los recursos individuales y colectivos" (p. 173). En este sentido, propone el análisis de la vulnerabilidad de los adultos mayores desde estudios longitudinales (historias de vida y encuestas) y a escala de hogar, abandonando limitadas aproximaciones basadas solo en el individuo. Este estudio expone la necesidad de generar estrategias de afrontamiento, para posponer los efectos negativos ambientales, sociales e individuales de la vejez, junto con disminuir el grado de vulnerabilidad de la población adulta mayor. Otorga importancia a los relatos de las personas investigadas, en lo referente a sus experiencias de vida y cómo significan estos acontecimientos, debido a que cuentan con un bagaje histórico que condiciona su manera de apreciar y de responder ante los estímulos externos.

En síntesis, si bien puede existir consenso respecto de que las personas mayores podrían ser más vulnerables que otros grupos etarios ante situaciones de desastre, debido a sus características demográficas asociadas a salud, movilidad y dependencia, es necesario ampliar el análisis y considerar que factores ambientales, tales como geografía del lugar en que habitan, material de construcción de las viviendas y en el caso específico de esta investigación, vegetación abundante de los cerros, también pueden tener incidencia en sus circunstancias de vulnerabilidad. Factores que son propios de países en desarrollo, como el nuestro, que obliga a las personas a habitar sectores alejados y en situaciones de 
habitabilidad precaria, producto entre otras cuestiones, del alto costo de vivir en sectores cercanos a los centros urbanos. De allí la relevancia de conocer desde sus relatos, cómo las personas mayores significan sus experiencias en situaciones de desastre.

\section{Teoría de la continuidad, un enfoque sociocultural de la vejez}

Este enfoque ha sido acuñado por B. L. Neugarten $(1961,1968)$ y R. Atchley (1969, 1971, 1993), enfatizando en la construcción social de la vejez. Dentro de sus postulados principales propone que "las personas realizan una serie de elecciones adaptativas a lo largo de la etapa adulta y del envejecimiento que suponen una continuación de los patrones de comportamiento mantenidos a lo largo de sus vidas" (Alcalde \& Laspeñas en Giró, 2005, p. 5). Por lo tanto, no existiría una ruptura radical ni transición brusca entre la edad adulta y la tercera edad, ni tampoco una visión cronológica ni de etapas vitales predeterminadas en cómo se comprende a las personas mayores, sino más bien una continuidad entre etapas. La vejez sería entendida como una prolongación de experiencias, proyectos y hábitos de vida del pasado y las personas al envejecer aprenderían a utilizar diversas estrategias de adaptación, que les ayudan a reaccionar eficazmente ante los sufrimientos y las dificultades de la vida. Atchley (1971) plantea que las personas deben mantener un equilibrio entre continuidad y cambio en condiciones internas y externas de su vida. De esta forma, en lo interno hace referencia a la estructura psicológica basada en recuerdos, vivencias, experiencias, ideas, valores, ideales, preferencias, que denotan la orientación de alguna conducta en particular. Con relación a la continuidad externa, las personas rememoran y actúan de acuerdo a sus patrones de interacción con otros, sus niveles de participación social y patrones sociales. La perspectiva de análisis que propone esta teoría revaloriza la voz del sujeto que vivencia el tránsito de adultez a vejez, otorgándole protagonismo a la expresión de sus experiencias, desde sus interpretaciones y significaciones, destacando la capacidad de aprendizaje que éste tiene para desenvolverse en distintas etapas y escenarios de su vida: lo personal, lo familiar, lo social. Por tanto, se diferencia de otras configuraciones teóricas como la biomédica, el enfoque de desarrollo del ciclo vital o la teoría de la desvinculación, que aprecian el envejecimiento como un fenómeno que puede estudiarse de manera aislada e independiente del significado que el sujeto le atribuye, como también ajeno a la incidencia de variables socioculturales. De allí el interés de este estudio de sustentarse desde la teoría de la continuidad, con el fin de recoger experiencias personales y sociales de manera inductiva, sin establecer patrones de conducta ni categorizaciones cronológicas ni generalistas, muchas veces centradas solo en el deterioro que puede implicar la vejez, dejando poco espacio a una consideración sociocultural de la misma. Por tanto, se amplía la mirada y se incorporan elementos de análisis desde la consideración de la subjetividad de los sujetos que la vivencian en interacción con los contextos socioculturales en que se desenvuelven. 
Significados sociales, una comprensión social del conocer

Continuando con la perspectiva de análisis desde la construcción social, según lo propuesto por Gergen (2006), los significados pueden entenderse como

las formas de interpretar y comprender el mundo, y la realidad social de los sujetos, incluyendo sus creencias y valores, los cuales son construidos socialmente en la interacción con otros, situados en un contexto histórico y cultural, que es exteriorizado a través de una serie de argumentaciones, discursos y premisas, que connotan las acciones de los individuos (p. 75).

El construccionismo social es una corriente teórica que nace en la segunda mitad del siglo XX, en pleno auge de la posmodernidad, donde uno de sus principales exponentes es el psicólogo social norteamericano Kenneth Gergen, quien ejecutó un ejercicio crítico de desconstrucción de los fundamentos epistemológicos y ontológicos de la psicología social tradicional, instaurando las bases para el desarrollo de una nueva perspectiva psicosociológica, ligada a la metáfora de la construcción (Sandoval, 2004, pp. 105-112). Es decir, ocurre un cambio de la mirada explicativa causal sobre el conocimiento del ser humano, hacia una nueva perspectiva relativista, donde su objeto de estudio son las relaciones con el mundo (Cañón, Peláez, \& Noreña, 2005, p. 240). El construccionismo social se interesa en cómo se generan los conceptos y su utilización, a través de las convenciones lingüísticas, construcciones sociales o culturales. Este proceso de análisis "deconstructivo" (Gergen, 1985, p.270), incorpora la historicidad, resaltando la relevancia del contexto cultural e histórico para los marcos de referencia interpretativos, a través de los cuales las personas otorgamos significado a lo que nos sucede.

Gergen (1996) afirma que los juegos del lenguaje, en los cuales emergen las verdades compartidas por una comunidad tienen un valor funcional, es decir, coordinar a los diferentes sujetos en torno a visiones construidas sobre la realidad. En este sentido, indica que "la verdad parece ser una cuestión de perspectivas, y éstas productos de intercambios y consensos sociales, es decir, construidas en los sistemas de comunicación social” (p. 99). La realidad es, a fin de cuentas, un conjunto de significados conversacionales socialmente compartidos. Destaca el lenguaje que se utiliza para explicar la acción humana en su relación con los otros, manifestándose por medio de los relatos, es por ello que el lenguaje en sí mismo no sirve de nada, hasta que el otro hace algo con él, de este modo se construyen las significaciones por medio de la comunicación e interacción con el otro. Gergen (1999) plantea que "la función del lenguaje es favorecer las relaciones y crear un código común para construir sentido" (p. 100) y, del mismo, modo Bruner (1991) afirma que "el lenguaje permite a los sujetos participar en la cultura, entender y construir sus significados” (p. 15). 
Para este estudio cuestionar las verdades aceptadas, tener en cuenta la especificidad histórica y cultural del conocimiento, y considerar que las interacciones sociales sustentan el conocimiento son premisas planteadas por Gergen (1985, pp. 270-275) que resultan necesarias al momento de fundamentar cómo las personas significan las experiencias que vivencian. Con relación a la primera, es necesario cuestionar la creencia de que el conocimiento convencional se basa solo en la observación objetiva e imparcial de la realidad, el construccionismo social recomienda escepticismo a la hora de considerar las propias ideas, sobre el mundo o su apariencia. Es decir, las verdades aceptadas en la sociedad no necesariamente corresponden a lo real, entonces la realidad es subjetiva, las personas aprehenden el mundo según sus experiencias de vida, donde la verdad de uno no necesariamente corresponde a la verdad de otro, es por esto que cada uno, construye sus propios significados respecto de sus vivencias.

En segundo lugar, es preciso tener en cuenta la especificidad histórica y cultural del conocimiento. Desde este principio, se busca conocer los significados tomando en consideración la historicidad y la cultura que rodea la vida de las personas y grupos sociales. A modo ilustrativo, la coyuntura económica y social, predominante en una cultura y una época determinada, produce una cierta concepción del mundo. Las personas construyen su conocimiento y significados a partir de la forma en que observan la realidad, a través del sistema cultural y social de un momento histórico determinado, respecto del cual pueden estar de acuerdo o no. Sin embargo, teñirá de todos modos los significados que elaboren. Finalmente, las interacciones sociales sustentan el conocimiento, es decir, el conocimiento es el resultado de una construcción colectiva; la verdad no deriva de la observación objetiva del mundo, sino de los procesos sociales y de las interacciones sociales. Desde esta perspectiva, es apreciable conocer cómo son y cómo ocurren las interacciones sociales de las personas con y en su entorno, y la manera en que tributan en el significado que éstos le otorgan a sus vivencias.

\section{METODOLOGÍA}

Paradigmáticamente, este estudio se sustentó desde el enfoque fenomenológico, con el fin de acceder a los significados que los individuos otorgan a su experiencia, desde sus propias interpretaciones (Vasilachis, 2006). Por lo anterior, la metodología utilizada fue cualitativa, buscando rescatar experiencias que han vivenciado las personas mayores a lo largo de su vida con respecto a situaciones de desastre, utilizando un enfoque biográfico, el cual según Pujadas (1992) "pretende mostrar el testimonio subjetivo de una persona, en que se recojan tanto los acontecimientos como las valoraciones que dicho sujeto, hace de su propia existencia, lo que se materializa en un relato autobiográfico obtenido por quien investiga mediante entrevistas" (p. 47).

El tipo de estudio fue exploratorio, con el fin de familiarizarse con este fenómeno relativamente desconocido y poco investigado. El diseño fue naturalista y sin control (Mella, 
2003), porque la recolección de datos ocurrió dentro del ambiente natural de los sujetos sin condicionar el contexto donde viven, lo que favoreció que se desenvolvieran de manera espontánea y cómoda. La técnica seleccionada para la recogida de datos fue la entrevista en profundidad (Canales, 2006), enfatizando en aspectos biográficos (Flores, 2009) de los sujetos de estudio, con el fin de poder apreciar a través de sus relatos, sus experiencias vividas, creencias y valores, realizándola de forma individual a cada persona seleccionada, existiendo un conjunto de preguntas y temas a explorar que se refirieron a consultas sobre vulnerabilidad ante los desastres a partir del contexto; capacidad de respuesta ante los desastres a partir de sus experiencias previas y reestructuración de sus vidas a partir de las interacciones sociales, respondiendo a los objetivos específicos de este estudio. Para asegurar su validez y fiabilidad, siguiendo lo que propone Pérez (2002), el guión temático fue previamente sometido a juicio de expertos y se hizo una prueba piloto, antes de su aplicación definitiva. Cada entrevista efectuada fue grabada, previa autorización de las personas investigadas, mediante la puesta en marcha de un protocolo de consentimiento informado, con el fin de resguardar su dignidad, anonimato y confidencialidad, antes, durante y después de todo el proceso investigativo, el que fue previamente aprobado por el Comité de Ética Científico de la Universidad que efectuó este estudio. Las entrevistas fueron llevadas a cabo durante los meses de enero a marzo del año 2015, aplicando dos entrevistas a cada persona seleccionada en las nuevas viviendas habitadas por ellos en el cerro La Cruz.

Las personas investigadas correspondieron a cuatro, seleccionadas de acuerdo al muestreo intencionado propuesto por Salinas (2010, p. 90) y Martínez-Salgado (2012, p. 615), debido a sus posibilidades de ofrecer información profunda y detallada, sobre el asunto de interés para la investigación. Para ello de acuerdo a criterios de heterogeneidad, representatividad y accesibilidad, se seleccionaron: una persona mayor damnificado varón que viva solo, una persona mayor damnificada mujer que viva sola, una persona mayor damnificado varón que viva acompañado y una persona mayor damnificada mujer que viva acompañada, tal como se esquematiza usando el casillero tipológico propuesto por Miguel Valles (2003, p. 93). La justificación de la selección de ambos criterios, es decir, situación familiar, entendida como si se vive solo o acompañado al momento del incendio, y género, ya sea hombre o mujer, se sustenta en la revisión de la literatura analizada, donde investigadores como Cohen y Ahearn (1989), Tilly (2000), SENAMA (2009), Olivo y Piña (2011), Guajardo (2013), Caballero y Massad (2013), FACSO/FLACSO Chile/SENAMA (2013), Huenchuán (2014), Hartog (2014) y Ortega (2018), hacen referencia a las condiciones de aislamiento, abandono o dependencia familiar en que pueden encontrarse las personas mayores y, por tanto, resulta importante conocer cómo éstas podrían condicionar sus experiencias ante desastres. En complemento, el ser hombre o mujer cuando se está en la tercera edad, puede ser una variable importante de considerar que colabore o no en su actual condición de vulnerabilidad socioambiental. A decir de Ortega (2018), "cuando se juntan las condiciones, ser adulto mayor y mujer, se potencian doblemente estas explotaciones y la producción de desigualdad 
persistente se amplifica, así como las condiciones de pauperización de estas personas" (p. 219). A continuación, en la Tabla 1 se sintetiza la configuración de la muestra de acuerdo a los criterios descritos.

\begin{tabular}{|c|c|c|c|c|}
\hline \multirow[b]{2}{*}{ GÉNERO } & \multirow[t]{2}{*}{ Criterios } & \multicolumn{2}{|c|}{ SITUACIÓN FAMILIAR } & \multirow[b]{2}{*}{$\begin{array}{l}\text { Sub } \\
\text { Total }\end{array}$} \\
\hline & & $\begin{array}{c}\text { Persona mayor } \\
\text { damnificado que } \\
\text { viva solo/a }\end{array}$ & $\begin{array}{c}\text { Persona mayor } \\
\text { damnificado que } \\
\text { viva acompañado/a }\end{array}$ & \\
\hline MUJER & & 1 & 1 & 2 \\
\hline HOMBRE & & 1 & 1 & 2 \\
\hline Sub Total & & 2 & 2 & Total. 4 \\
\hline
\end{tabular}

Tabla 1. Muestra seleccionada de acuerdo a casillero tipológico de Miguel Valles, 2003.

El análisis de datos fue llevado a cabo mediante la técnica de contenido interpretativo elaborada por González Rey (2006), análisis que fue validado por un proceso de triangulación múltiple, es decir, se validaron los resultados con las personas investigadas; entre los integrantes del equipo investigador (triangulación intersubjetiva) y se dispuso de la triangulación de un experto.

\section{RESULTADOS}

Antes de desarrollar los resultados principales respecto de cómo las personas mayores significan su experiencia en situaciones de desastre, a partir del incendio ocurrido en Valparaíso, es necesario conocer el perfil social de cada una de ellas, lo que colaborará además en la comprensión de sus interpretaciones, subjetividades y relatos.

Entrevistada 1: Mujer de 70 años de edad, 20 años de residencia en el cerro La Cruz, trabajadora de casa particular, casada, separada de hecho, dos hijas. Cuando ocurrió el grave desastre incendiario vivía sola en una casa cedida ubicada en un terreno propiedad de su hermano, luego de éste recibió una solución habitacional definitiva en otro sector, sin embargo, continuó viviendo en el cerro La Cruz. Actualmente vive con su pareja.

Entrevistada 2: Mujer de 60 años de edad, 45 años de residencia en cerro La Cruz, dueña de casa, casada, tres hijos. Cuando ocurrió el siniestro vivía con su marido e hija en una vivienda de su propiedad. A la fecha de mayo de 2015, aún esperaba una solución 
habitacional definitiva. Continúa viviendo en el cerro en vivienda autoconstruida en compañía de su esposo.

Entrevistado 3: Hombre de 75 años de edad, reside en cerro La Cruz desde que nació, jubilado, casado, dos hijos. Cuando ocurrió el incendio vivía con su cónyuge en vivienda arrendada. Solucionó su problema de vivienda accediendo al subsidio habitacional, a raíz de esto, obtuvo un departamento en otro cerro de Valparaíso, sin embargo, prefirió quedarse en el cerro La Cruz. En el presente, vive con su esposa en casa de uno de sus hijos.

Entrevistado 4: Hombre de 94 años de edad, reside en cerro La Cruz desde hace 81 años, jubilado, viudo, padre de once hijos, de los cuales nueve fueron afectados por el incendio. En el momento del siniestro habitaba solo en vivienda propia. Solucionó su situación habitacional de manera independiente, optando por la autoconstrucción, sin recibir a la fecha de mayo de 2015 apoyo estatal. Actualmente vive con una hija.

\section{Vulnerabilidad ante los desastres a partir del contexto}

Las personas entrevistadas expresan que en su contexto persisten factores de riesgo que las hacen sentirse vulnerables a ser afectadas por otras situaciones, luego de ocurrido el incendio. La mayor preocupación es ser víctima de la delincuencia, producto de la disminución de vecinos que habitan en el sector en el tiempo inmediatamente posterior al siniestro, lo que ha provocado que valoren la importancia que tienen los vecinos, para revertir ese sentimiento de inseguridad generado por el abandono que expresan sentir en el presente. La interacción permanente con vecinos les devolvería la tranquilidad y seguridad que sentían antes del incendio, reforzando en las personas mayores el sentido de pertenencia y la historicidad sociocultural compartida en la vivencia cotidiana y que, según relatan, caracteriza a quienes habitan en el cerro La Cruz.

...hay mucho temor, porque hay muchos robos aquí en el cerro, muchos robos, entonces uno no puede salir poh. Si sale un vecino, no puede salir el otro, porque no podemos dejar solo (Entrevistado 3).

Yo no sé cuánto tiempo o cuántos años van a pasar, para que yo vuelva a sentir la tranquilidad que yo tenía. Yo ahora no tengo tranquilidad (Entrevistada 2).

Otro sentimiento de vulnerabilidad en que los entrevistados y las entrevistadas coinciden, se refiere a que el entorno físico después del incendio ha vuelto a presentar condiciones de riesgo que lo han tornado propicio para nuevos focos de este tipo (crecimiento indiscriminado de pasto, arbustos, malezas, escombros y desperdicios en quebradas y sitios eriazos). Esto 
se ve empeorado por la percepción que tienen de que sus vecinos no se preocupan por prevenir los riesgos de desastre, lo que produce en ellos sentimientos de desprotección y fragilidad. Al mismo tiempo que se advierte en sus testimonios, que externalizan totalmente la responsabilidad de tomar medidas de prevención solo en los otros, no identificando en ninguno de los cuatro testimonios analizados algún grado de responsabilidad personal, frente al riesgo de nuevos focos incendiarios que existen en el sector.

...sí, claro que sí, y ahora mismo, fíjate que con esto que pasó, la tierra se quemó, pensé que iba a costar más que saliera el pasto, por el contrario, hay pasto por todos lados, mucho... (Entrevistado 4).

...limpiando el entorno mío, yo no puedo limpiar lo de los demás, porque no se previenen también del pasto, que el pasto se pone seco y los incendios también es culpa de la gente...la gente es muy dejá, es muy dejá, porque uno se encarga de limpiar el lado de uno (Entrevistada 1).

Al referirse a sus condiciones de vulnerabilidad surge espontáneamente en las cuatro personas entrevistadas, la atribución de una valoración positiva de las experiencias vividas en el pasado, en el contexto del cerro descrito, expresando nostalgia al recordar las relaciones humanas que se establecían con los vecinos, caracterizadas por lazos de cooperación y amistad, que hoy en día, según ellas, serían distintas.

...no eran como vecinos, eran como familia, porque pasaban, se pedían apoyo, las mamás solucionaban problemas, además la gente era más humana, era más cariñosa, como que las cosas las marcaban a las personas, ahora no, es más indiferente, más fría... (Entrevistada 1).

Es recurrente en sus relatos la valoración positiva del pasado, en ocasiones casiidealizada. Las personas entrevistadas relatan con melancolía pasajes de sus vidas compartidas con los vecinos, incluso expresan la necesidad de refugiarse en sus recuerdos para no enfrentar la angustia del presente, ocasionada por la magnitud de la pérdida provocada por el incendio. Refieren que antiguamente las interacciones entre vecinos eran habituales y constructivas, desarrollándose la colaboración mutua entre quienes habitaban en el cerro, lo que contrastaría con la situación actual en que las dinámicas de interacción a nivel vecinal actualmente han disminuido y cambiado, dejando de lado la solidaridad de las prácticas comunitarias que eran características.

...me acuerdo que la gente del cerro de escasos recursos, fallecía alguien, la gente ayudaba en alguna cosa en la noche y acompañaban a las personas... (Entrevistado 3). 
Las personas entrevistadas también expresan una significación simbólica del cerro La Cruz, más allá del entorno físico, fundada en sus historias transcurridas en él, las que se vinculan con la crianza de los hijos, las relaciones familiares y las dinámicas sociales construidas en su contexto, que evocan en ellos sentimientos de añoranza. En este sentido, es preponderante destacar la significación que le otorgan al cerro La Cruz, desde el arraigo que tienen con el lugar, el que se manifiesta en los recuerdos que han construido, a lo largo de los años y que evidencian, se mantienen intactos luego del desastre; por consiguiente, a pesar de tener historias de vida diferentes, antes y después de lo ocurrido, están dispuestas y coinciden en mencionar que desean reconstruir sus viviendas y sus vidas en el cerro La Cruz.

(En referencia al ambiente del cerro La Cruz) ...es toda mi vida, es decir, yo aquí en el cerro, estoy acostumbrado acá (Entrevistado 4).

Este arraigo también se denota a nivel familiar, puesto que la totalidad de las personas investigadas significan el siniestro incendiario como una tragedia familiar, ya que afectó a varios miembros de sus familias que habitan en el sector, lo que manifiestan con desazón, debido al sentimiento de pérdida que expresan por las historias familiares construidas, así como los variados recuerdos albergados en común con el sector, lo que evidencia una vez más el apego a nivel personal y familiar, que mantienen con el cerro y que es transmitido, a las generaciones venideras, pérdida material y simbólica, que es comprendida como un detrimento incalculable para las personas entrevistadas y sus familiares, respecto del sentido que le otorgan a este hecho en sus vidas.

...nos criamos todos los hermanos juntos y éramos tantos hermanos, y siempre en el mismo cerro y antes había más armonía...la gente se hablaba más, porque la gente era más cariñosa (Entrevistada 1).

Capacidad de respuesta ante los desastres a partir de sus experiencias previas

En relación a la valoración que le asignan a su capacidad de respuesta, surgen al menos tres elementos necesarios de considerar en el análisis. El primero de ellos dice relación con su reacción inmediata en el momento del siniestro caracterizada por una incredulidad de lo que estaba ocurriendo y de un miedo que como emoción los paralizó en la acción e inundó su visión de mundo en los días siguientes.

...claro que me sentía segura, pero jamás pensé que iba a pasar una cosa así, nunca y esta casa como era de concreto pensé que se iba a guarecer, lo mío...no, para mí no, nunca imaginé que a nosotros se nos iban a quemar todas las casas... (Entrevistada 1). 
(En referencia al momento del incendio en su casa) “...me asusté cuando el bombero me dijo, no si no hay solución para esto, lo material se recupera y yo no, y de ahí que yo no quería bajar, mi hermano no le hacía caso, le gritaba y no no... (Entrevistado 3).

...ustedes tienen que comprender que a mí me andaban trayendo, a mí y a tu papá, pa' todos lados, me llevaban para allá, me llevaban para acá, nosotros no nos dábamos cuenta (Entrevistada 2).

Esta primera reacción paralizante de miedo fue progresivamente moderándose, gracias al apoyo familiar, de vecinos y voluntarios, hasta transformarse en una actitud resiliente fundada en una valoración positiva respecto de sí mismos y del reconocimiento de sus propias capacidades para hacer frente al desastre incendiario y sus consecuencias. No obstante, la emoción de miedo permanece en uno de los tres casos investigados en la actualidad. Según las cuatro personas entrevistadas, el estrés postraumático no habría sido atendido profesionalmente en tiempos oportunos, es decir, de manera inmediatamente posterior al incendio ni tampoco después, según se les habría ofrecido por instancias gubernamentales.

...si yo siento miedo...cuando siento a las bombas y es algo eléctrico en mi cuerpo, me da como un tirón y yo quedo así, como paralizada... (Entrevistada 1).

...me va a creer que yo me siento aquí y a veces me corren las lágrimas, no soy apega' a lo material, pero le cuesta a uno tener las cosas, le cuesta, además; cuando uno tiene tantos hijos eh, uno le da prioridad a los hijos y la casa va quedando así, va quedando... cuando ya los hijos están un poquito más grandes, ya uno puede terminar la casa (Entrevistada 2).

...hay adultos mayores que se han muerto porque no soportaron de ver que se quemó su casa y se murieron, cayeron al hospital y murieron (Entrevistado 3).

Las personas estudiadas significan unánimemente el incendio como uno de los sucesos más graves de su vida, para algunas incluso como el evento más trágico. Emerge de manera natural en sus relatos, comparar la gravedad del incendio con los terremotos de Valparaíso de 1985 y 2010, desastres naturales que para las cuatro sería significado como menos grave y peligroso que un incendio, además de no involucrar pérdida material absoluta de sus bienes físicos ni de sus bienes subjetivos o inmateriales como recuerdos familiares.

...con el terremoto las casas no se vienen abajo, son de madera, se pueden menear, ladear, pero no se caen, tendría que ser un socavón, un hoyo por abajo; pero el incendio 
se llevó todo, no quedó nada, no nos quedó ninguna foto, la ropa, todas las cosas que habían adentro, los televisores, todo se quemaron, no quedo nada, quedo solo un montón de escombros, no más... (Entrevistado 4).

...porque en un terremoto pasa todo tan rápido, uno se asusta, pero queda el movimiento, pero uno está confiada que nada pasa, pero esto del incendio fue tremendo, ... un terremoto yo encuentro que no es nada comparado a un incendio porque el incendio no respeta nada, las llamas oh... ese día (Entrevistada 1).

Otro elemento relevante de analizar en este eje dice relación con las diferencias de género que pueden observarse en la muestra respecto de cómo significan el evento mismo del incendio, después de ocurrido éste. De esta forma, se denota una coincidencia por parte de las mujeres entrevistadas, al significar desde la religiosidad el desastre como una prueba u obstáculo más que deben sobrellevar, apoyándose en su fe en Dios, lo que podría comprenderse desde su socialización en algún credo religioso que tendría incidencia en su cosmovisión de mundo y que podría ser característico de este grupo etario, si se le analiza generacionalmente. Entonces su capacidad de respuesta y los sucesos de sus vidas estarían, según sus testimonios, fuera de ellas, es decir, mediados o conducidos por un ente externo de carácter superior.

...sí, no he vivido cosa más terrible que, Dios quiera que nunca más pase, todas las noches siempre rezo, por todos, mi familia, yo lo veo por todos mis hermanos, por toda mi familia... un terremoto yo encuentro que no es nada comparado a un incendio (Entrevistada 2).

...Dios todavía nos tiene aquí y eso que hubo 16 muertos, y podríamos ser nosotros, yo creo que hay mucha gente que estaba muy mal antes y ahora está mejor... (Entrevistada 1).

En cambio, para los varones entrevistados, su significación del incendio es realizada a partir de experiencias de vida, en las que habrían desarrollado actitudes y conductas de resiliencia, que pese al desconcierto y a la significación del siniestro como una desgracia, los motivan a sobreponerse y seguir adelante. Esto podría estar amparado también en una explicación generacional, si se estima que los sujetos de la muestra varones se encuentran entre los 75 a 94 años, lo que habla de hombres socializados bajo el modelo hegemónico patriarcal, donde la responsabilidad de ser líderes de familia, padres y proveedores, es reconocible. Por ello, a diferencia de las mujeres de la muestra, su capacidad de respuesta estaría dentro de ellos, conducida por ellos mismos, desde una visión antropocéntrica más que teocéntrica. 
...no, yo creo que los años nos han dado esa sabiduría de siempre salir adelante, con lo poco, ella trabajaba, yo ya estaba jubilado, así que todo eso, hay que tirar para arriba no más (Entrevistado 3).

...yo quería levantar una casa, para después demostrarle a mis abuelos que están muertos ya, que eso lo vuelvo a levantar para mis nietos y para los bisnietos que vienen... (Entrevistado 4).

...no, si no hemos perdido la alegría nosotros, decíamos primero, no teníamos tiempo de pensar de lo que habíamos pasado, se nos quemó todo, pero no podíamos echarnos a morir, yo no podía echarle la culpa a ella, ni ella a mí, porque son cosas que pasaron... (Entrevistado 3).

\section{Reestructuración de sus vidas a partir de las interacciones sociales}

En este eje de análisis destaca que tres de las personas investigadas tienen una significación positiva del entorno familiar, debido a las dinámicas de colaboración y apoyo permanentes que mantienen, simbolizando la familia como un pilar fundamental en la reestructuración de sus vidas para enfrentar y sobreponerse a la compleja experiencia vivida.

...mi familia siempre ha estado preocupada, mi hermano al que se le quemó la casa, siempre me trae ropa o alguna cosa, todas las cuatro hermanas somos unidas, pero todo esto es por mi mamá, siempre fue así para todos por igual...y uno piensa en todo lo que se llevó el incendio, todo el sacrificio... (Entrevistada 1).

Sin embargo, en este eje también se aprecian diferencias de género en la estimación de apoyo familiar, cuando una entrevistada significa la familia únicamente como la proyección del matrimonio que construyó junto a su cónyuge, puesto que relata no recibir apoyo de su familia, especialmente de sus hijos; expresa sentirse incomprendida, en situación de abandono y desprotección. Fragilización que podría vincularse a su condición de persona mayor y mujer.

...nosotros no tenemos apoyo de los hijos, eh... nosotros los criamos, se enamoraron, se fueron, se casaron, ellos tienen su propia familia, ellos se preocupan muy poco de nosotros, eso fue algo terrible, eso fue lo que me dio el golpe final (Entrevistada 2).

Se evidencia en este relato una búsqueda constante de apoyo en personas externas a su familia, significándolas como soportes que la orientaron y motivaron, animándola a sobreponerse y a seguir adelante, es por ello que le otorga especial importancia a la ayuda 
que tuvo de los voluntarios y otras agrupaciones, para reconstruir su vida.

...Yo he tenido mucho apoyo de gente que no es nada mío, de gente desconocida, mucho apoyo, mucho amor, cariño, han estado en los momentos más difíciles, han estado conmigo, no lo he esperado yo de mi familia, pero de gente extraña, me ha dado mucho apoyo y cariño, y eso ha sido muy fundamental para mí, porque realmente lo necesitaba, yo no conté con mi familia para nada (Entrevistada 2).

De modo similar, las otras tres personas significan a los voluntarios como una primera ayuda fundamental, para reorganizarse y comenzar a reconstruir sus vidas, expresando sentimientos de agradecimiento, debido a que indican que ellos fueron los primeros en auxiliarlos, entregando, además, ánimo y acompañamiento en los momentos posteriores a dicho desastre.

...la gente llegó por montones aquí y venía un mar de gente por Avenida Francia, niños de colegio, toda la gente y traían cosas más encima, venían a ayudar y aquí pasaban viendo qué necesitábamos, y la ayuda al tiro, estuvieron unos niños extranjeros, eran americanos, y vinieron a ayudar acá, con gente de acá y como estamos en la pasada, fue fácil que nos ayudaran... (Entrevistada 1).

Lo que sí, nosotros estamos muy agradecidos, muy agradecidos, que no hayamos cómo pagarle, son de los universitarios... ellos se pusieron la camiseta y subían los cerros desde abajo, cargados de comida, bidones de agua, con herramientas para ayudar a limpiar los escombros... gracias a la voluntad ayudaron a levantar casas (Entrevistado 4).

En referencia a la valoración que le otorgaron a la ayuda entregada por las instituciones públicas, se produce una dicotomía, ya que dos de los entrevistados, que no habitaban en una vivienda propia al momento del desastre incendiario, fueron beneficiados con una solución habitacional definitiva. Por esta razón, significan el apoyo de las instituciones como positivo expresando gratitud, ya que contribuyó a que visualizaran el futuro de manera más esperanzadora y tranquila. Sin embargo, expresan que no fue de su total conformidad que dicha solución habitacional fuese la entrega e instalación de una mediagua en el mismo sitio, donde vivían antes del incendio.

...si no tengo nada que decir, para comer nos dieron como tres a cuatros meses, todos preocupados, incluso tengo una foto con la Michelle Bachelet... (Entrevistada 1).

Por el contrario, las otras dos personas que eran propietarias al momento del incendio, significan este apoyo de manera negativa, como insuficiente y desorganizado, expresando 
inconformidad y frustración, sentimientos que emergen desde su desesperanza aprendida, por lo que en ambos casos la solución a su situación habitacional fue dada por ellas mismas, desde la estrategia de autoconstrucción. Es importante señalar que, en referencia a este apoyo, la totalidad de las personas mayores entrevistadas significó como un obstáculo en la reestructuración de sus vidas, la burocracia de las instituciones públicas, que no habrían hecho distinción de edad para priorizar la entrega de ayuda. Expresan una valoración negativa de lo que denominan "exceso de trámites" para acceder a algunos beneficios. En complemento, también critican cómo al mediano plazo, esta colaboración se fue extinguiendo hasta desaparecer por completo, antes del término del mismo año del incendio.

Ahora, con el incendio se vino al suelo... muy devastador. Aquí en este lado que yo vivo, son todos propietarios y no les han construido todavía, porque hay muchos que optaron por irse, eh... aceptaron el subsidio y se fueron, porque no quisieron volver a empezar nuevamente (Entrevistada 2).

...se fue mucho tiempo, no vinieron para nada, no vinieron, eh... nosotros pensábamos que pudieran venir para la pascua y darnos una alegría, que fue una pascua muy triste, muy triste la pascua que pasamos, nosotros aquí en el cerro, la gente toda se unió aquí, prendíamos velitas y todos llorábamos, todos llorábamos el año nuevo igual (Entrevistado 4).

Otro significado compartido por las personas entrevistadas es la valoración simbólica de la vivienda, más allá de un bien material, comprendiéndola como una construcción socio histórico-familiar, pudiendo interpretarse desde los sujetos que su historicidad y experiencia se relacionan con la subjetividad y el intercambio social desarrollado antiguamente en el cerro. Significando además la vivienda desde lo simbólico, identificándola como el espacio social donde desarrollaron sus vidas e historia, identificando varios hitos relevantes de su biografía familiar en dichas viviendas: el matrimonio, el nacimiento de los hijos, la instalación de las viviendas de los hijos en el mismo sitio, el nacimiento de los nietos, entre otros. De allí que muchos de ellos ante la solución habitacional ofrecida por los organismos públicos, de trasladarse a vivir a otras comunas de la región, optaron por desecharla, prefiriendo quedarse en el cerro y construir nuevas viviendas. Esto es relevante de analizar si se considera que, en el sector investigado y en el propio Valparaíso, es característico que en un mismo espacio (sitio) vivan varios integrantes de un mismo clan familiar (padres- hijos; hermanos).

...no sé qué, fíjate que perdí todas las cosas de los matrimonios que se habían hecho arriba, había vivido mi hijo, había vivido una nieta, un nieto, eso fue después que mi madre murió...son cosas que no se pueden recuperar, la cama que me había hecho mi padre...pucha, son muchos recuerdos... (Entrevistado 3). 


\section{CONCLUSIONES}

A partir del análisis interpretativo, es preciso concluir respecto de la presencia de significados que colaboran en comprender cómo las personas mayores vivencian situaciones de desastre, desde su experiencia en el incendio de Valparaíso de abril de 2014, respondiendo al objetivo central de esta investigación. De esta forma, afloran en el análisis indicios que permiten cuestionar ciertas verdades aceptadas o estereotipadas respecto de la vejez, al mismo tiempo que coexisten algunas certidumbres vinculadas a las condiciones de desigualdad e invisibilidad en que viven algunas personas mayores en nuestro país, que las posicionan en un escenario de vulnerabilidad socioambiental, asociado a variables estructurales por sobre su fisonomía demográfica.

De acuerdo a los testimonios de las cuatro personas entrevistadas, el contexto investigado continuaría siendo propicio para la ocurrencia de futuros siniestros, tales como incendios y aluviones, puesto que el análisis revela que históricamente no existiría una cultura de prevención a nivel local, denotado desde la falta de organización vecinal y la ausencia de apoyo de estamentos especializados para la prevención y afrontamiento de estas situaciones. De esta forma, persistirían condiciones de riesgo que podrían llevar a que se genere una nueva tragedia, debido al material de las viviendas y a su ubicación en zonas boscosas y de difícil acceso. Esto emerge y se aprecia en los resultados, lo que nos lleva a comprender y reafirmar que los desastres son producto de procesos sociales históricamente construidos de acumulación de riesgos que afectan con mayor fuerza a quienes presentan precarias condiciones socioeconómicas y habitan en la periferia de las ciudades, aumentando la desigualdad y en este caso generando nuevos escenarios de aislamiento y exclusión social para quienes viven la tercera edad. De allí que sentimientos de desamparo y desprotección son recurrentes en los relatos estudiados, expresando una constreñida relación de dependencia con otros: familia, vecinos, voluntarios y especialmente Estado, representado este último en organismos locales y delegaciones presidenciales. Catalogado como ausente, desde valoraciones de abandono y desilusión, debido al retiro progresivo y total del acompañamiento al primer semestre de producido el incendio.

La capacidad de respuesta ante situaciones de desastres, en este estudio, es significada desde dos polos de un mismo continuo que colaboran en desmitificar aquella construcción social que estigmatiza y relega a las personas mayores a una posición de desvalorización, dependencia y minusvalía como lo revelan numerosas investigaciones en Chile. En este aspecto, las personas investigadas reconocen que la valoración de sí mismas y de su situación fue inicialmente negativa, tiñéndose de emociones de miedo y frustración, durante el incendio y posterior a él, debido a la magnitud de las pérdidas sufridas a nivel personal, familiar y social, especialmente en esta etapa de sus vidas. Sin embargo, en un segundo momento, transcurrido el siniestro, desde la consideración de la reestructuración de sus vidas a partir de las interacciones sociales, se sintieron fortalecidos por el apoyo de familiares y voluntarios, 
alcanzando una apreciación positiva de su propia capacidad de respuesta, significada como una actitud resiliente originada en sus experiencias, lo que expresa la continuidad de tránsitos internos y externos que ellas han vivenciado a lo largo de sus vidas. En síntesis, le atribuyen un alto valor a la experiencia adquirida, en situaciones previas de riesgos naturales y sociales, logrando desplegar una adecuada capacidad para resolverlos, para organizar y administrar recursos individuales y colectivos.

La referencia a los criterios muestrales enriquece en este nivel las conclusiones, puesto que la condición de ser hombre y ser mujer efectivamente imprimió diferencias en cómo se significa esta experiencia. No obstante, una limitación de este estudio fue la imposibilidad de ahondar en ello, lo que al mismo tiempo abre una proyección de futuras investigaciones que lo profundicen. De esta forma, las mujeres investigadas expresaron mayor fragilidad y dependencia al momento de enfrentar el siniestro, lo que podría aumentar el riesgo de exclusión en ellas, en comparación a los varones, resaltando la necesidad de profundizar en factores asociados a la feminización de la población mayor, no solo comprendida como un indicador numérico, sino referida a mayores condiciones de desigualdad en la vejez, debido a la desigualdad histórica que comporta ser mujer en nuestro país, que se representa en bajos índices de incorporación al mercado laboral precarizando ingresos y seguridad social en conjunto con la atribución casi exclusiva a labores de cuidado en la familia, desde infantes, enfermos y otras personas mayores. Sin embargo, en este estudio tanto hombres como mujeres lograron transitar a una condición de empoderamiento en su capacidad de resolución de este evento traumático y en la conducción de sus vidas. En complemento, la consideración de vivir solo o acompañado al momento del incendio es apreciable, no obstante, para las personas investigadas, lo es más, estar solo o acompañado en las vivencias posteriores al siniestro, donde emerge la figura familiar como el gran pivote desde donde se sostienen y desde donde ellos sostienen sus clanes familiares, especialmente en el caso de los varones, en este último aspecto. En general, las personas investigadas no expresan condiciones de abandono familiar, salvo un caso, sino más bien manifiestan poseer un patrimonio de lazos familiares, con hermanos, hijos y nietos. La configuración familiar que habita los cerros de Valparaíso refiere a varias familias de una misma raíz genealógica ocupando un mismo sitio y compartiendo cotidianamente un mismo espacio físico y relacional. Por lo tanto, las personas mayores damnificadas de este estudio serían capaces de generar las herramientas necesarias para sobreponerse gracias a valores, costumbres y relaciones sociales que han ido construyendo a lo largo de sus vidas, en su cotidianeidad e interacción con otros, siendo la interacción con la familia la más estimada.

La capacidad de respuesta de los servicios gubernamentales es significada espontánea y recurrentemente por las personas mayores investigadas como burocrática, tardía y no afín con sus expectativas, lo que implicó para ellas un refuerzo de su propia capacidad de toma de decisiones para resolver lo que identifican como la mayor pérdida de sus vidas. Refieren estar conscientes de que quizás no tengan tiempo suficiente para disfrutar sus viviendas 
reconstruidas y en algunos casos autoconstruidas, pero que, a pesar de aquello, sus hijos sí podrán hacerlo, frente a lo que manifiestan consuelo y satisfacción personal. Se aprecia una prolongación de experiencias, proyectos y hábitos de vida del pasado, capacidades de aprendizaje y adaptación, que les ayudan a reaccionar eficazmente ante los sufrimientos y las dificultades vividas.

La alta valoración otorgada a la vivienda, como construcción simbólica de la historia familiar; el sentido de pertenencia al sector en que se habita y las interacciones sociales que propician la colectividad construida por décadas, incluso desde la infancia, con vecinos y familiares, según la población investigada, no serían elementos considerados en las soluciones habitacionales ofrecidas, generando desconcierto y emoción de desarraigo cuando se ofrece individual y no comunitariamente el traslado a comunas alejadas de Valparaíso, lo que fragiliza sus estrategias para hacer frente a esta situación, colabora en la ruptura de los vínculos sociales y propicia solo la responsabilidad individual. En complemento, si bien la baja disposición de recursos económicos les obliga a vivir en los cerros, resulta ser para ellos una indignidad que las soluciones ofrecidas hayan sido viviendas de emergencia o que, a más de un año del incendio, algunos no tuviesen una solución habitacional definitiva.

La consideración de las subjetividades de las personas mayores debe ser parte de un dispositivo mayor, de una cultura de responsabilidad social, donde las problemáticas públicas, en este caso socioambientales no se resuelvan solo desde el engranaje de las interacciones sociales, la potenciación de la responsabilidad individual o la iniciativa de privados. De allí que constituye una urgencia considerar su experiencia en el establecimiento de estrategias de prevención y afrontamiento de situaciones de desastre en los ámbitos local y central. Si bien el incendio no destruyó, sino más bien reafirmó vínculos sociales, empoderamiento y sentido de arraigo a sus contextos espaciales, territoriales y familiares, este estudio deja entrever mecanismos desde distintos agentes sociales (la cultura, el Estado, la familia) que podrían posicionar a las personas mayores en situaciones de riesgo y maltrato estructural, más allá de su semblanza estadística e imaginarios sociales de dependencia, carga e inutilidad. Romper con la omisión de estas subjetividades podría posicionarnos como una cultura que asume la denuncia y transformación necesarias para dejar de situar, nosotros mismos, a las personas mayores como grupo vulnerable y vulnerado.

\section{REFERENCIAS}

AMUCH. (2017). Los Adultos Mayores en las comunas de Chile: actualidad y proyecciones. Santiago de Chile: Asociación de Municipalidades de Chile.

Arcila, P., Jaramillo, J., Mendoza, Y., \& Cañón, O. (2009). Comprensión del significado desde Vygotsky, Bruner y Gergen. Diversitas: Perspectivas en Psicología, 6(1), 37-49.

Atchley, R. C. (1993). Continuity Theory and the Evolution of Activity in Later Adulthood en J. R. Kelly (Ed.), Activity and Aging: Staying involved in later life. Newbury Park: Sage. 
Atchley, R. C. (1971). Retirement and leisure participation: Continuity or crisis? The Gerontologist, 11(1), 13-17.

Atchley, R. C. (1969). A Continuity Theory of normal aging. The Gerontologist, 2, 183-190.

Bruner, J. (1991). The Narrative Construction of Reality. Critical Inquiry, 18(1), 1-21.

Caballero, M., \& Massad, C. (2013). Antecedentes en torno al maltrato hacia las personas mayores en Chile En M. Abusleme \& G. Guajardo (Eds.), El maltrato hacia las personas mayores en la Región Metropolitana, Chile. Investigación cualitativa en vejez $y$ envejecimiento (pp. 21-32). Santiago de Chile: Ediciones SENAMA. CASEN.

Canales, M. (2006). Metodologías de Investigación Social: Introducción a los Oficios. Santiago de Chile: Ediciones Lom.

Cañón, O., Peláez, M., \& Noreña N. (2005). Reflexiones sobre el socioconstruccionismo. Psicología, 1(2), 238-245.

CELADE (2006). Manual sobre indicadores de calidad de vida en la vejez. Chile: Centro Latinoamericano y Caribeño de Demografía CELADE - División de Población de la CEPAL.

Cohen, R. E., \& Ahearn, F. L. Jr. (1989). Manual de la atención de Salud Mental para víctimas de Desastres. Ciudad de México: HARLA.

Cutter, S. L., Boruff, B. J., \& Lynn, W. S. (2003). Social Vulnerability to Environmental Hazards. Social Science Quarterly. 84(2), 242-261.

Flores, R. (2009). Observando Observadores: Una Introducción a las Técnicas Cualitativas de Investigación Social. Santiago de Chile: Ediciones Universidad Católica de Chile.

Formiga, N., Prieto, M. B., \& Medus, S. (2009). Vulnerabilidad, Accesibilidad y Calidad de Vida. Los adultos mayores en la ciudad de Bahía Blanca. Estudios sobre Geografía y Análisis Espacial con sistemas de Información Geográfica (GESIG). Programa de Estudios Geográficos (PROEG), 1(1), 37-61

Gergen, K. (1985). The Social Constructionist Movement in Modern Psychology. American Psychologist. 40(3), 266-275.

Gergen, K. (1996). Realidades y Relaciones. Aproximaciones a la construcción social. Barcelona: Editorial Paidós.

Gergen, K. (1999). An Invitation to Social Construction. London: Sage.

Gergen, K. (2006). Realidades y Relaciones: Aproximación a la Construcción Social. Barcelona: Editorial Paidós Básica.

Giró, J. (2005). Envejecimiento, Salud y Dependencia. La Rioja: Universidad de La Rioja. Biblioteca de investigación No42.

González Rey, F. (2006). Investigación Cualitativa y Subjetividad. Guatemala C.A.: Oficina de Derechos Humanos del Arzobispado de Guatemala.

Guajardo, G. (2013). Una tipología de las significaciones y sentidos sociales sobre el maltrato desde la mirada de las personas mayores. En M. T. Abusleme \& G. Guajardo (Eds.), El maltrato hacia las personas mayores en la Región Metropolitana, Chile (pp. 127-138). Santiago de Chile: Ediciones SENAMA- Flacso. 
Hartog, J. (2014). Disaster resilience in an ageing world: how to make policies and programmes inclusive of older people. London: Helpage International (HIA).

Huenchuán, S. (2014). El maltrato hacia las personas mayores: conceptos, normas y experiencias de políticas en el ámbito internacional. En M. T. Abusleme \& M. Caballero (Eds.), Maltrato a las personas mayores en Chile. Haciendo visible lo invisible. Santiago de Chile: Ediciones SENAMA.

IFRC. (2014). ¿Qué es un desastre? Recuperado de: http://www.ifrc.org/es/introduccion/ disaster-management/sobre-desastres/que-es-un desastre/ Consulta: 10/10/2014.

López-Ibor, J. J. (2004). ¿Qué son desastres y catástrofes? Actas Españolas de Psiquiatría, 32(2), 1-16.

Martínez-Salgado, C. (2012). El muestreo en investigación cualitativa. Principios básicos y algunas controversias. Ciencia \& Saúde Coletiva, 17(3), 613-619.

Mella, O. (2003). Metodología Cualitativa en Ciencias Sociales y Educación. Santiago de Chile: Editorial Primus.

Neugarten, B. L. (1989). Teoría de Continuidad. Barcelona: Editorial Paidós.

Neugarten, B. L. (1968). Adult Personality. Toward a psychology of the life cycle, en Neugarten, B. L. y Tobin, S. S. (Ed.): Middle age and aging. Chicago: The University of Chicago Press. Neugarten, B. L., Havighurst, R. J., \& Tobin S. S. (1961). The measurement of life satisfaction. Journal of Gerontology, 16, 134-143.

Observatorio de Valparaíso (2015). Reconstrucción Gran Incendio de Valparaíso. Reporte1. Estado de Avance de la Reconstrucción. Recuperado de: www.observatoriovalparaiso.cl

Olivo, M., \& Piña, M. (2011). Envejecimiento y Cultura en IberoAmérica. Talca: Universidad Católica del Maule, Universidad Central, Universidad Veracruzana.

ONU. (1982). Plan de Acción Internacional de Viena sobre el Envejecimiento. Primera Asamblea Mundial sobre el Envejecimiento: Viena.

Ortega, D. (2018). Envejecimiento y trato hacia las personas mayores en Chile: una ruta de la desigualdad persistente. Sophia Austral, 22(2), 223-246.

Paola, J., Danel, P., \& Manes, R. (2012). Reflexiones en torno al Trabajo Social en el campo Gerontológico. Tránsitos, miradas e interrogantes. Buenos Aires: Universidad de Buenos Aires.

Pérez, G. (2002). Investigación Cualitativa. Retos e Interrogantes, Tomo II: Técnicas. Madrid: Editorial La Muralla, S.A.

Pérez, S. (2013). La Vulnerabilidad Social antes y después de los desastres socio naturales: del impacto ambiental al devenir subjetivo. Chile: Centro de Investigación en Vulnerabilidades y Desastres Socionaturales, Universidad de Chile.

PNUD. (2014). Plan de recuperación post desastre y transición al desarrollo de la comuna de Valparaíso, un enfoque participativo y de reducción del riesgo de desastres. Santiago de Chile: Programa de las Naciones Unidas para el Desarrollo.

Pujadas, J. M. (1992). El método biográfico: el uso de las historias de vida en Ciencias Sociales. 
Madrid: Centro de Investigaciones Sociológicas.

Salinas, D. (2010). ¿A cuántos y quiénes preguntar? Una aproximación al muestreo cuantitativo y cualitativo en investigación social y educacional. Valparaíso: Ediciones Universitarias de Valparaíso, Pontificia Universidad Católica de Valparaíso.

Sánchez, D. G., \& Egea, C. J. (2011). Enfoque de vulnerabilidad social para investigar las desventajas socio ambientales. Su aplicación en el estudio de los adultos mayores. Papeles de población, 17(69), 151-185.

Sandoval, J. (2004). Representación, discursividad y acción situada. Primera Edición. Valparaíso: Editorial Universidad de Valparaíso.

SECPLA (2014). Borrador de Diagnóstico Municipal para la Reconstrucción. Valparaíso: Secretaría Comunal de Planificación, Ilustre Municipalidad de Valparaíso.

SENAMA (2009). Las personas mayores en Chile: Situación, avances y desafíos del envejecimiento y la vejez. Santiago de Chile: Servicio Nacional del Adulto Mayor.

SENAMA/FACSO/FLACSO Chile (2013). Encuesta Inclusión y exclusión social de las personas mayores en Chile. Opiniones, percepciones, expectativas y evaluaciones. Tercera encuesta nacional de inclusión y exclusión social. FACSO, FLACSO Chile, SENAMA.

Skarwar, F. (2014). Incendio forestal en España causado por factores naturales y agravados por la acción del hombre. Recuperado de: http://www.bomberosmijas.com/archivos/ planes_leyes/incendios_forestales_espana.pdf Consulta: 10/10/2014.

Tilly, Ch. (2000). La desigualdad persistente. Buenos Aires: Manantial.

Valles, M. (2003). Técnicas cualitativas de investigación social: reflexión metodológica y práctica profesional. Madrid: Editorial Síntesis.

Vargas, J. E. (2002). Políticas públicas para la reducción de la vulnerabilidad frente a los desastres naturales y socio-naturales. Santiago de Chile: División de Medio Ambiente y Asentamientos Humanos, CEPAL.

Vasilachis de Gialdino, I., Ameigeiras, A., Chernobilsky, L., Giménez V., Mallimaci, F., Mendizábal, N., Neiman, G.,... \& Sonería, A. (2006). Estrategias de Investigación Cualitativa. Barcelona: Gedisa. 